\title{
Sub-classification of Advanced-Stage Hepatocellular Carcinoma: A Cohort Study Including 612 Patients Treated with Sorafenib
}

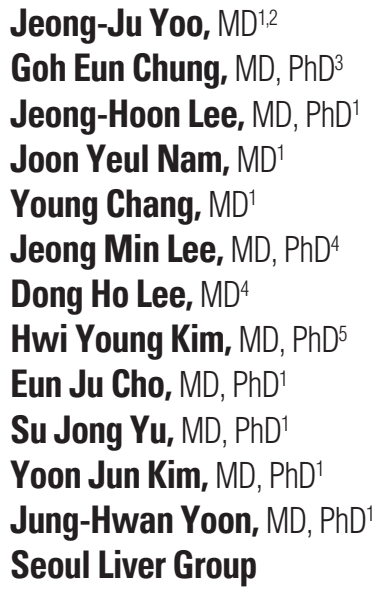

${ }^{1}$ Department of Internal Medicine and Liver Research Institute, Seoul National University College of Medicine, Seoul, ${ }^{2}$ Department of Gastroenterology and Hepatology, Soonchunhyang University Bucheon Hospital, Bucheon, ${ }^{3}$ Department of Internal Medicine, Healthcare Research Institute, Gangnam Healthcare Center, Seoul National University Hospital, Seoul, "Department of Radiology, Seoul National University Hospital, Seoul National University College of Medicine, Seoul, ${ }^{5}$ Department of Internal Medicine, Ewha Womans University School of Medicine Liver Center, Ewha Womans University Mokdong Hospital, Seoul, Korea

Correspondence: Jeong-Hoon Lee, MD, PhD Department of Internal Medicine and Liver Research Institute, Seoul National University Hospital, 101 Daehak-ro, Jongno-gu, Seoul 03080, Korea

Tel: $82-2-2072-2228$

Fax: 82-2-762-9662

E-mail: pindra@empal.com

Received March 10, 2017

Accepted April 25, 2017

Published Online May 15, 2017

*Jeong-Ju Yoo and Goh Eun Chung contributed equally to this work.

\section{Purpose}

Advanced hepatocellular carcinoma (HCC) is associated with various clinical conditions including major vessel invasion, metastasis, and poor performance status. The aim of this study was to establish a prognostic scoring system and to propose a sub-classification of the Barcelona-Clinic Liver Cancer (BCLC) stage C.

\section{Materials and Methods}

This retrospective study included consecutive patients who received sorafenib for BCLC stage C HCC at a single tertiary hospital in Korea. A Cox proportional hazard model was used to develop a scoring system, and internal validation was performed by a 5 -fold cross-validation. The performance of the model in predicting risk was assessed by the area under the curve and the Hosmer-Lemeshow test.

\section{Results}

A total of 612 BCLC stage $C$ HCC patients were sub- classified into strata depending on their performance status. Five independent prognostic factors (Child-Pugh score, $\alpha$-fetoprotein, tumor type, extrahepatic metastasis, and portal vein invasion) were identified and used in the prognostic scoring system. This scoring system showed good discrimination (area under the receiver operating characteristic curve, 0.734 to 0.818 ) and calibration functions (both $p<0.05$ by the Hosmer-Lemeshow test at 1 month and 12 months, respectively). The differences in survival among the different risk groups classified by the total score were significant ( $p<0.001$ by the log-rank test in both the Eastern Cooperative Oncology Group 0 and 1 strata).

\section{Conclusion}

The heterogeneity of patients with $B C L C$ stage $\mathrm{CHCC}$ requires sub-classification of advanced HCC. A prognostic scoring system with five independent factors is useful in predicting the survival of patients with BCLC stage C HCC.

\section{Key words}

Hepatocellular carcinoma, Advanced-stage, Sorafenib, Prognosis 


\section{Introduction}

Hepatocellular carcinoma (HCC) is the sixth most common cancer and has the second cancer-related death rate worldwide [1]. In most cases, HCC develops in patients with underlying chronic liver disease such as cirrhosis and hepatitis $B$ or $C$ virus infection [2,3]. To reduce the mortality from HCC, a surveillance program applied in patients at high risk for developing HCC [4]. However, more than half of all HCC patients are diagnosed at an unresectable stage, which time there are limited treatment options and a low chance of cure $[5,6]$. The international clinical practice guidelines have endorsed the Barcelona Clinic Liver Cancer (BCLC) staging system for HCC classification and treatment strategy [7-9]. However, most HCC patients present with BCLC stage $\mathrm{C}$ at the time of diagnosis [1]. According to current guidelines, sorafenib is the only proven systemic treatment option for improving the overall survival of patients with BCLC stage C HCC. However, sorafenib provides only a modest survival benefit of 2 to 3 months compared to placebo [10].

BCLC stage C HCC encompasses heterogenous subgroups that show differences in vascular invasion, extrahepatic spread and performance status. Moreover, BCLC stage C HCC includes various tumor types such as nodular, monofocal, or infiltrating tumors, and is thus, associated with wide differences in treatment responses and survival. Therefore, sub-classification of BCLC C is required to predict the precise prognosis and offer more effective therapeutic strategies [11]. For example, the Hong Kong Liver Cancer system, which was recently developed based on Asian patients, recommend surgical resection for a subgroup of BCLC C HCC patients [12]. Therefore, the aims of this study was to refine a prognostic scoring system using independent factors as well as to propose a sub-classification for BCLC stage C HCC based on a large cohort.

\section{Materials and Methods}

\section{Study population}

This retrospective study included consecutive patients who received sorafenib for BCLC stage C HCC between January 2008 and December 2014 at a single tertiary hospital (Seoul National University Hospital, Seoul, Korea). The diagnosis of HCC was based on noninvasive criteria or pathologic examination according to the guidelines of the American Association for the Study of Liver Disease or European Association for the Study of the Liver $[7,8]$.
Table 1. Baseline characteristics

\begin{tabular}{|c|c|}
\hline Characteristic & No. $(\%)(n=612)$ \\
\hline Age, mean \pm SD (yr) & $60.4 \pm 10.9$ \\
\hline $\log (A F P)$, mean $\pm S D$ & $5.6 \pm 3.5$ \\
\hline \multicolumn{2}{|l|}{ Sex } \\
\hline Male & $515(84.2)$ \\
\hline Female & $97(15.8)$ \\
\hline \multicolumn{2}{|l|}{ Etiology } \\
\hline $\mathrm{HBV}$ & $490(80.0)$ \\
\hline $\mathrm{HCV}$ & $43(7.0)$ \\
\hline Alcohol & $32(5.2)$ \\
\hline Other & $47(7.7)$ \\
\hline \multicolumn{2}{|l|}{ Child-Pugh class } \\
\hline A & $463(75.7)$ \\
\hline B & $128(20.9)$ \\
\hline $\mathrm{C}$ & $21(3.4)$ \\
\hline \multicolumn{2}{|l|}{ Tumor type } \\
\hline Nodular & $498(81.4)$ \\
\hline Infiltrative & 114 (18.6) \\
\hline \multicolumn{2}{|l|}{ ECOG PS } \\
\hline 0 & $443(72.4)$ \\
\hline 1 & $169(27.6)$ \\
\hline \multicolumn{2}{|l|}{ PV invasion } \\
\hline No & $359(58.7)$ \\
\hline Yes & $253(41.3)$ \\
\hline \multicolumn{2}{|l|}{ Metastasis } \\
\hline No & $160(26.1)$ \\
\hline Yes & $452(73.9)$ \\
\hline
\end{tabular}

SD, standard deviation; AFP, $\alpha$-fetoprotein; HBV, hepatitis B virus; HCV, hepatitis C virus; ECOG PS, Eastern Cooperative Oncology Group performance status; PV, portal vein.

The baseline information, including demographic profiles, laboratory findings and the etiology of liver disease was obtained at the time of HCC diagnosis. Radiologic images were reviewed by two independent radiologists (J.M.L. and D.H.L.) with $>10$ years of experience, who were unaware of the clinical information of the study patients. In cases of discordance, a third independent and experienced radiologist reviewed the images and a consensus was achieved among the three radiologists. The study was approved by the Institutional Review Board of the Seoul National University Hospital; the requirement for informed consent was waived.

\section{Statistical analysis}

The primary endpoint was overall survival which was measured from the date of the first sorafenib treatment to the date of death from any cause. Death registration data from the Korea National Statistical Office were used. The censored 
Table 2. Multivariate analysis of prognostic factors related to survival

\begin{tabular}{lcccc} 
& Coefficient & HR & $95 \%$ CI & p-value \\
Child-Pugh score & 0.301 & 1.352 & $1.266-1.444$ & $<0.001^{*}$ \\
Log(AFP) & 0.107 & 1.113 & $1.082-1.145$ & $<0.001^{*}$ \\
Infiltrative tumor & 0.509 & 1.664 & $1.299-2.130$ & $<0.001^{*}$ \\
PV invasion & 0.160 & 1.174 & $0.930-1.483$ & 0.178 \\
Metastasis & 0.114 & 1.121 & $0.882-1.424$ & 0.349 \\
\hline
\end{tabular}

$\mathrm{HR}$, hazard ratio; CI, confidence interval; AFP, $\alpha$-fetoprotein; PV, portal vein. ${ }^{*} \mathrm{p}<0.05$.

date was defined as last date of follow-up, or data cutoff date (i.e., 30 November 2016).

We used the Student's t test to compare continuous variables and the chi-square test or Fisher exact test to compare categorical variables. We used an analysis of variance to compare continuous variables. The Kaplan-Meier method with log-rank test was used to compare overall survival between groups. The Cox proportional hazard model was used to establish a scoring model for predicting the prognosis of HCC patients.

To assess the discrimination capacity, we calculated the area under receiver operating characteristic (AUROC) curves at each time point. In addition, we conducted internal validation of the scoring system using a 5-fold cross-validation procedure with the Hosmer-Lemeshow test $[13,14]$.

Statistical analysis was performed using SPSS ver. 19.0 (IBM Corp., Armonk, NY) and R language ver. 3.2.0 (R Foundation for Statistical Computing, Vienna, Austria). p-values less than 0.05 were considered statistically significant.

\section{Results}

\section{Patients}

Among 756 patients who received sorafenib for HCC between 2008 and 2014, 612 (81.0\%) were classified as BCLC stage $C$ and were included in this study. Baseline characteristics are shown in Table 1. The mean age of the patients was $60.3 \pm 10.9$ years and $84.2 \%$ were male. Approximately threefourths of patients had underlying chronic hepatitis B. Portal vein invasion, infiltrative tumor type, and extrahepatic metastasis were present in $41.3 \%, 18.6 \%$, and $73.9 \%$ of participants, respectively. Most patients were heavily treated for HCC according to the advice of the physician and the preference of the patient. Among them, 82 patients received sorafenib as a first line treatment for HCC. The treatment modalities prior to
Table 3. Scoring system

\begin{tabular}{|c|c|}
\hline Risk factor & Point \\
\hline \multicolumn{2}{|l|}{ Child-Pugh score } \\
\hline $5-6$ & 0 \\
\hline 7-9 & 7 \\
\hline \multicolumn{2}{|l|}{$\log (\mathrm{AFP})$} \\
\hline $0-3$ & 0 \\
\hline $3-6$ & 3 \\
\hline 6-9 & 6 \\
\hline$>9$ & 10 \\
\hline \multicolumn{2}{|l|}{ Tumor type } \\
\hline Nodular & 0 \\
\hline Diffuse or infiltrative & 5 \\
\hline \multicolumn{2}{|l|}{ Portal vein invasion } \\
\hline Absent & 0 \\
\hline Present & 1 \\
\hline \multicolumn{2}{|l|}{ Extrahepatic metastasis } \\
\hline Absent & 0 \\
\hline Present & 1 \\
\hline Total points & 24 \\
\hline
\end{tabular}

AFP, $\alpha$-fetoprotein.

sorafenib were as follows: 475 patients with transarterial chemoembolization, 188 with surgical resection, 101 with radiofrequency ablation, 82 with percutaneous ethanol injection, 111 with radiotherapy, and 55 with systemic cytotoxic chemotherapy.

\section{Derivation of the prognostic model}

Because the proportional hazards assumption was rejected for the Eastern Cooperative Oncology Group (ECOG) performance status, the ECOG performance status was determined as strata: ECOG 0 or 1 . In the multivariate analysis, five independent prognostic factors (Child-Pugh score, $\alpha$-fetoprotein [AFP], tumor type [nodular vs. diffuse/infiltrative types], 
Table 4. Results of internal validation at each time point

\begin{tabular}{lcccc} 
& 1 Month & 3 Months & 6 Months & 12 Months \\
AUC & 0.818 & 0.759 & 0.738 & 0.734 \\
p-value of Hosmer-Lemeshow test & $0.031^{*}$ & 0.122 & 0.125 & $0.002^{*}$ \\
\hline
\end{tabular}

AUC, area under time-dependent receiver operating characteristic curve. ${ }^{*} \mathrm{p}<0.05$.
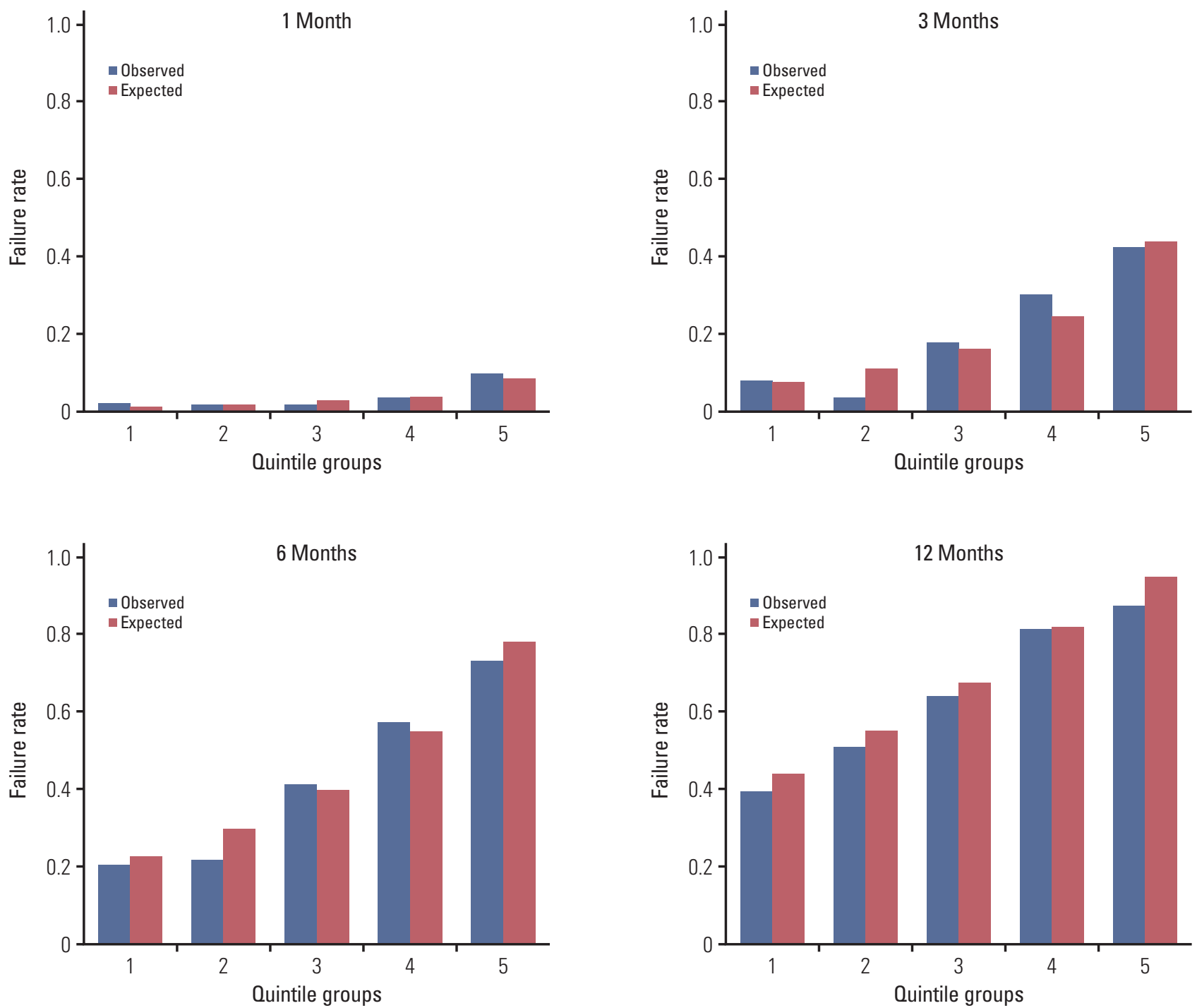

Fig. 1. The results of internal validation using 5-fold cross-validation at each time point.

extrahepatic metastasis, and portal vein invasion) were identified (Table 2). The proportional hazards assumption was not rejected for these five variables utilizing coefficients derived from multivariable analysis, a point scoring system was developed (Table 3 ). The scoring system assigned from 1 to 10 points for the presence of each factor, resulting in a total score that ranged from 0 to 24 . 
A
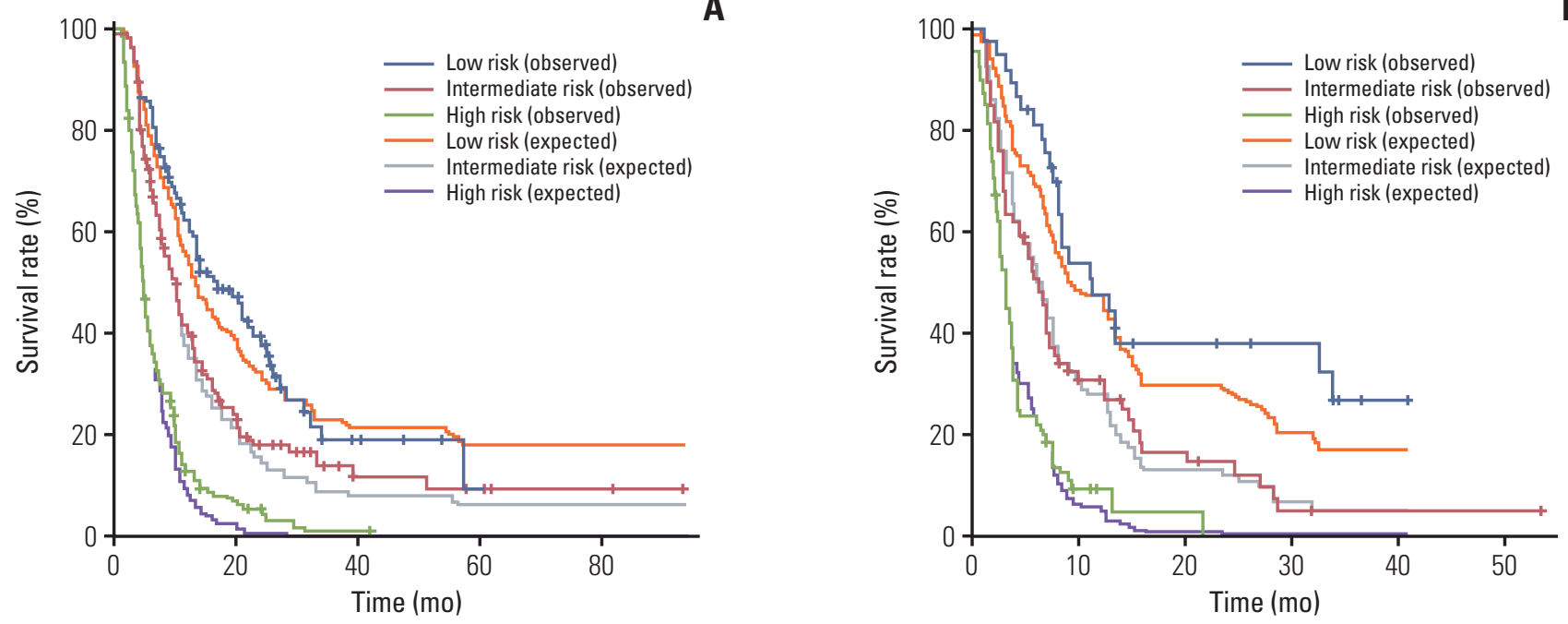

$\begin{array}{rrrrrrrr}\text { No. at risk (mo) } & 0 & 1 & 3 & 6 & 12 & 24 & 36 \\ \text { Low } & 150 & 105 & 99 & 83 & 56 & 22 & 7 \\ \text { Intermediate } & 180 & 178 & 161 & 114 & 64 & 21 & 10 \\ \text { High } & 158 & 151 & 107 & 57 & 18 & 6 & 2\end{array}$

$\begin{array}{rrrrrrrr}\text { No. at risk (mo) } & 0 & 1 & 3 & 6 & 12 & 24 & 36 \\ \text { Low } & 37 & 37 & 36 & 30 & 16 & 9 & 4 \\ \text { Intermediate } & 71 & 71 & 50 & 36 & 18 & 7 & 2 \\ \text { High } & 61 & 54 & 32 & 15 & 3 & - & -\end{array}$

Fig. 2. Kaplan-Meier analysis of the cumulative overall survival rate according to the risk group. (A) Eastern Cooperative Oncology Group performance status (ECOG PS) 0. (B) EGOG PS 1.

Table 5. Median overall survival in each risk group

\begin{tabular}{|c|c|c|c|c|}
\hline & Median OS (mo) & Interquartile range & Hazard ratio & p-value \\
\hline \multicolumn{5}{|l|}{ ECOG PS 0} \\
\hline Low & 16.73 & $7.2-30.70$ & 1 & $<0.001$ \\
\hline Intermediate & 9.57 & $4.67-19.20$ & 1.622 & \\
\hline High & 4.50 & $2.57-9.13$ & 3.598 & \\
\hline \multicolumn{5}{|l|}{ ECOG PS 1} \\
\hline Low & 11.27 & 7.30-not estimated & 1 & $<0.001$ \\
\hline Intermediate & 6.30 & $2.70-14.17$ & 2.228 & \\
\hline High & 3.23 & $1.87-4.60$ & 4.685 & \\
\hline
\end{tabular}

p-value by log-rank test among three groups. OS, overall survival; ECOG PS, Eastern Cooperative Oncology Group performance status.

\section{Validation of the scoring system}

We performed the temporal internal validation for the scoring system. As a result, this scoring system showed good discrimination (AUORC, 0.734 to 0.818 ) (Table 4 ) and calibration functions by 5 -fold cross-validation ( $\mathrm{p}=0.030$ at 1 month and $\mathrm{p}=0.002$ at 12 months by the Hosmer-Lemeshow test, respectively) (Fig. 1).

According to these results, we classified patients with BCLC
C HCC into three groups according to their scores: the lowrisk group $(<4$ points), intermediate-risk group (4 to 11 points), and high-risk group (> 11 points). The three risk groups demonstrated significantly different survival rates in patients with ECOG status of 0 and 1 (both $p<0.001$ by the log-rank test) (Fig. 2). Among patients with ECOG 0, the median overall survival of the low-, intermediate-, and highrisk groups were 16.7, 9.6, and 4.5 months, respectively. Among patients with ECOG 1, the median overall survival of 
the low-, intermediate-, and high-risk groups were 11.3, 6.3, and 3.2 months, respectively (Table 5).

\section{Discussion}

We developed a points-based scoring system to predict the prognosis of BCLC stage C HCC patients who received sorafenib treatment in the Korean population based on the five independent variables and stratified by ECOG performance status: the Child-Pugh score, AFP, tumor type, extrahepatic metastasis, and portal vein invasion. Using this scoring system, BCLC stage C HCC patients can be stratified into three risk groups, with good discrimination performance and calibration function. To assess its robustness, we performed internal validation. As a result, the overall survival was hierarchized by our sub-classification. The median overall survival was 4.5 months in the high risk group while it was 16.7 months in the low risk group for EGOG 0, suggesting that BCLC C HCC is a heterogeneous group with markedly different prognosis.

Because the coefficients were differentially weighted to predict the prognosis among variables such as the ChildPugh score, extrahepatic metastasis and portal vein invasion, which are components for classifying BCLC stage, it is better to sub-classify BCLC C HCC than to classify these patients into the same group. Interestingly, the tumor type, AFP level, Child-Pugh score and ECOG performance status showed strong effects on prognosis rather than portal vein invasion and metastasis and this situation was reflected in our scoring system. Although the important role of the tumor type on the prognosis of HCC has been reported [15], the tumor type has not been adapted as a variable in many staging systems [16-18]. However, the prognostic implication of the tumor type was confirmed and reflected in our scoring system. In addition, the baseline hepatic function defined as the ChildPugh score and tumor biology, defined as the AFP level were essential for predicting the prognosis of patients with BCLC C HCC and were included in our scoring system.

Most variables included in our scoring system were similar to those in previous studies. In the CLIP staging, the ChildPugh score, tumor morphology, AFP level, and portal vein thrombosis were defined as prognostic factors [19]. A systematic review showed the most robust predictors of HCCrelated death are portal vein thrombosis, tumor size, the AFP level and Child-Pugh score [20]. Recently, a study performed in France established a new score system for stratify BCLC stage C HCC using five independent prognostic values [21]. These five parameters included in the scoring system were the Child-Pugh score, performance status, AFP level, number of nodules and infiltrative nature of the tumor. However, the number of patients with BCLC stage C HCC in the test cohort was relatively small $(n=161)$ in this previous study [21]. In contrast, the current study was based on a large cohort of 612 patients treated with sorafenib for BCLC C stage HCC.

The characteristics of the study population are crucial for identifying a scoring system that can predict prognosis. In our study, the proportion of infiltrative tumor type was $18 \%$, as observed in another study [22], while nearly 50\% of HCC cases constituted infiltrative tumors in other studies [21,23]. Infiltrative HCC shows different clinical and radiological features compared to nodular HCC [24], and these features are difficult to detect, especially in the presence of background cirrhosis. Infiltrative HCC also carries a worse prognosis than nodular HCC because of the tumor burden in combination with vascular invasion, the presence of extrahepatic metastasis, a high serum AFP level and symptoms at presentation [15]. In our scoring system, the infiltrative tumor type was assigned 5 points, suggesting a critical role for the tumor type in predicting the prognosis.

Portal vein thrombosis is an important clue diagnosing of infiltrative HCC with a high association of up to $68 \%-100 \%$ [25]. Portal vein thrombosis may develop as a primary feature of infiltrative HCC [26]. In autopsy series, it was reported that up to $44 \%$ of $\mathrm{HCC}$ cases have portal vein thrombosis [27]. In our study, $41 \%$ of the patients showed portal vein invasion, and the presence of portal vein invasion achieved 1 point in the scoring system. A recent study reported that HCC patients with portal vein thrombosis have heterogeneous prognosis with respect to the serum AFP levels [28], indicating that portal vein invasion is a comprehensive variable that can be influenced by multiple factors instead of a single, simple variable.

The prognostic and diagnostic value of AFP is well known in HCC patients $[17,18]$. Poor differentiation, vascular invasion, and a large tumor size have significant associations with high AFP levels [29]. A recent study proposed the incorporation of AFP into the sub-classification of BCLC C stage HCC patients, suggesting AFP may serve as a potential sub-classification factor [30]. In our scoring system, the AFP level was scored from 0 to 10 points and our results confirming AFP as a marker for predicting the prognosis of BCLC stage C HCC.

There are several potential limitations in this study. First, the patients in the study underwent treatment with multiple modalities prior to sorafenib treatment. However, to minimize potential bias, we only selected patients who were treated with sorafenib, which is the currently recommended treatment option in patients with BCLC stage C HCC. Second, we investigated patients at a single institution and did not perform external validation. Thus, an external validation study performed at multiple facilities is needed to confirm 
our scoring system.

In conclusion, the heterogeneity of BCLC stage C HCC patients indicates the need for the sub-classification of advanced HCC. The prognostic scoring system described herein incorporates five independent factors and is useful for predicting the survival of patients with BCLC stage $\mathrm{C} \mathrm{HCC}$.

\section{Conflicts of Interest}

Conflict of interest relevant to this article was not reported.

\section{Acknowledgments}

This study was funded by grant from the National R\&D Program for Cancer Control, Ministry for Health and Welfare, Republic of Korea (1420050); Seoul National University Hospital Research Funds (03-2016-0380 and 06-2016-2880); and a grant from Liver Research Foundation of Korea as part of Bio Future Strategies Research Project.

\section{References}

1. Park JW, Chen M, Colombo M, Roberts LR, Schwartz M, Chen PJ, et al. Global patterns of hepatocellular carcinoma management from diagnosis to death: the BRIDGE Study. Liver Int. 2015;35:2155-66.

2. El-Serag HB. Hepatocellular carcinoma. N Engl J Med. 2011;365:1118-27.

3. Sherman M. Hepatocellular carcinoma: epidemiology, surveillance, and diagnosis. Semin Liver Dis. 2010;30:3-16.

4. Chan AC, Poon RT, Ng KK, Lo CM, Fan ST, Wong J. Changing paradigm in the management of hepatocellular carcinoma improves the survival benefit of early detection by screening. Ann Surg. 2008;247:666-73.

5. Llovet JM, Bruix J. Systematic review of randomized trials for unresectable hepatocellular carcinoma: Chemoembolization improves survival. Hepatology. 2003;37:429-42.

6. Llovet JM, Di Bisceglie AM, Bruix J, Kramer BS, Lencioni R, Zhu AX, et al. Design and endpoints of clinical trials in hepatocellular carcinoma. J Natl Cancer Inst. 2008;100:698-711.

7. Bruix J, Sherman M; Practice Guidelines Committee, American Association for the Study of Liver Diseases. Management of hepatocellular carcinoma. Hepatology. 2005;42:1208-36.

8. European Association for the Study of the Liver; European Organisation for Research and Treatment of Cancer. EASLEORTC clinical practice guidelines: management of hepatocellular carcinoma. J Hepatol. 2012;56:908-43.

9. Forner A, Llovet JM, Bruix J. Hepatocellular carcinoma. Lancet. 2012;379:1245-55.

10. Llovet JM, Ricci S, Mazzaferro V, Hilgard P, Gane E, Blanc JF, et al. Sorafenib in advanced hepatocellular carcinoma. N Engl J Med. 2008;359:378-90.

11. Cabibbo G, Enea M, Attanasio M, Bruix J, Craxi A, Camma C. A meta-analysis of survival rates of untreated patients in randomized clinical trials of hepatocellular carcinoma. Hepatology. 2010;51:1274-83.

12. Yau T, Tang VY, Yao TJ, Fan ST, Lo CM, Poon RT. Development of Hong Kong Liver Cancer staging system with treatment stratification for patients with hepatocellular carcinoma. Gastroenterology. 2014;146:1691-700.

13. Efron B, Tibshirani RJ. An introduction to the bootstrap. Lon- don: Chapman \& Hall/CRC; 1994.

14. Hosmer DW Jr, Lemeshow S. The multiple logistic regression model: applied logistic regression. New York: John Wiley \& Sons; 1989. p. 25-37.

15. Yopp AC, Mokdad A, Zhu H, Mansour JC, Balch GC, Choti $\mathrm{MA}$, et al. Infiltrative hepatocellular carcinoma: natural history and comparison with multifocal, nodular hepatocellular carcinoma. Ann Surg Oncol. 2015;22 Suppl 3:S1075-82.

16. Llovet JM, Bru C, Bruix J. Prognosis of hepatocellular carcinoma: the BCLC staging classification. Semin Liver Dis. 1999;19:329-38.

17. Okuda K, Ohtsuki T, Obata H, Tomimatsu M, Okazaki N, Hasegawa $\mathrm{H}$, et al. Natural history of hepatocellular carcinoma and prognosis in relation to treatment: study of 850 patients. Cancer. 1985;56:918-28.

18. Lei HJ, Chau GY, Lui WY, Tsay SH, King KL, Loong CC, et al. Prognostic value and clinical relevance of the 6th Edition 2002 American Joint Committee on Cancer staging system in patients with resectable hepatocellular carcinoma. J Am Coll Surg. 2006;203:426-35.

19. A new prognostic system for hepatocellular carcinoma: a retrospective study of 435 patients: the Cancer of the Liver Italian Program (CLIP) investigators. Hepatology. 1998;28:751-5.

20. Tandon P, Garcia-Tsao G. Prognostic indicators in hepatocellular carcinoma: a systematic review of 72 studies. Liver Int. 2009;29:502-10.

21. Adhoute X, Penaranda G, Raoul JL, Blanc JF, Edeline J, Conroy G, et al. Prognosis of advanced hepatocellular carcinoma: a new stratification of Barcelona Clinic Liver Cancer stage C: results from a French multicenter study. Eur J Gastroenterol Hepatol. 2016;28:433-40.

22. Benvegnu L, Noventa F, Bernardinello E, Pontisso P, Gatta A, Alberti A. Evidence for an association between the aetiology of cirrhosis and pattern of hepatocellular carcinoma development. Gut. 2001;48:110-5.

23. Kneuertz PJ, Demirjian A, Firoozmand A, Corona-Villalobos C, Bhagat N, Herman J, et al. Diffuse infiltrative hepatocellular carcinoma: assessment of presentation, treatment, and outcomes. Ann Surg Oncol. 2012;19:2897-907. 
24. Demirjian A, Peng P, Geschwind JF, Cosgrove D, Schutz J, Kamel IR, et al. Infiltrating hepatocellular carcinoma: seeing the tree through the forest. J Gastrointest Surg. 2011;15: 2089-97.

25. Sereni CP, Rodgers SK, Kirby CL, Goykhman I. Portal vein thrombus and infiltrative HCC: a pictoral review. Abdom Radiol (NY). 2017;42:159-70.

26. Shah ZK, McKernan MG, Hahn PF, Sahani DV. Enhancing and expansile portal vein thrombosis: value in the diagnosis of hepatocellular carcinoma in patients with multiple hepatic lesions. AJR Am J Roentgenol. 2007;188:1320-3.

27. Pirisi M, Avellini C, Fabris C, Scott C, Bardus P, Soardo G, et al. Portal vein thrombosis in hepatocellular carcinoma: age and sex distribution in an autopsy study. J Cancer Res Clin
Oncol. 1998;124:397-400.

28. Carr BI, Guerra V. Low alpha-fetoprotein levels are associated with improved survival in hepatocellular carcinoma patients with portal vein thrombosis. Dig Dis Sci. 2016;61:937-47.

29. Liu C, Xiao GQ, Yan LN, Li B, Jiang L, Wen TF, et al. Value of alpha-fetoprotein in association with clinicopathological features of hepatocellular carcinoma. World J Gastroenterol. 2013;19:1811-9.

30. Zhang N, Gu J, Yin L, Wu J, Du MY, Ding K, et al. Incorporation of alpha-fetoprotein into subclassification of BCLC C stage hepatocellular carcinoma according to a 5-year survival analysis based on the SEER database. Oncotarget. 2016;7: 81389-401. 